Research Article

\title{
Lipidomics and Anti-Inflammation Activity of Brown Algae, Lobophora sp., in Vietnam
}

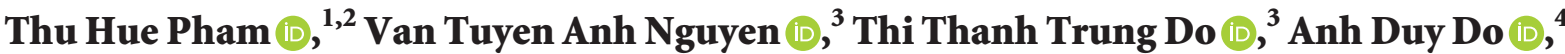 \\ Duc Tien Dam $\mathbb{D}^{5},{ }^{5}$ Thi Thanh Van Tran $\mathbb{D}^{6},{ }^{6}$ Quoc Long Pham $\mathbb{D},{ }^{3}$ and Tat Thanh Le $\left.\mathbb{D}\right)^{1,3}$ \\ ${ }^{1}$ Graduate Univesity of Science and Technology, Vietnam Academy of Science and Technology, Hanoi, Vietnam \\ ${ }^{2}$ Vietnam Naval Academy, Nha Trang, Vietnam \\ ${ }^{3}$ Institute of Natural Products Chemistry, Vietnam Academy of Science and Technology, Hanoi, Vietnam \\ ${ }^{4}$ Research Institute for Marine Fisheries, Hai Phong, Vietnam \\ ${ }^{5}$ Institute of Marine Environment and Resources, Vietnam Academy of Science and Technology, Hanoi, Vietnam \\ ${ }^{6}$ Nhatrang Institute of Technology Research and Application, Vietnam Academy of Science and Technology, Hanoi, Vietnam \\ Correspondence should be addressed to Tat Thanh Le; thanh.biotech@gmail.com
}

Received 2 October 2020; Revised 23 November 2020; Accepted 27 November 2020; Published 9 December 2020

Academic Editor: Andrea Mastinu

Copyright ( 92020 Thu Hue Pham et al. This is an open access article distributed under the Creative Commons Attribution License, which permits unrestricted use, distribution, and reproduction in any medium, provided the original work is properly cited.

Lobophora sp., belonging to brown macro algae phylum, is found in coral reefs. In this study, the fatty acid composition, lipid classes, polar lipid molecular forms, and bioactivities of this algae have been determined. It follows that five classes including polar lipid (Pol), sterol (ST), free fatty acids (FFA), triacylglycerol (TAG), and hydrocarbon and wax (HW), 23 fatty acids containing 5 PUFAs (ALA, GLA, AA, EPA, and DHA) and 157 molecular types of polar lipid group containing 48 phospholipid molecular forms belonging to 4 subclasses (PI (11), PC (14), PG (22), PA (1)), 45 glycolipid molecular forms classified into 3 subclasses of MGDG (8), DGDG (1), SQDG (36), and 64 betaine lipid molecular forms belonging to 2 subclasses (DGTA (37), DGTS (27)) have been identified for the first time from this algae. Furthermore, both polar lipid (PL) and unpolar lipid (UPL) show the NO inhibition activities with values of $\mathrm{IC}_{50}$ ranging from 52.10 to $66.21 \mu \mathrm{g} / \mathrm{mL}$. Thus, lipid of this brown algae could promise to be a potential source for application in food, cosmetic, and pharmaceutic industry.

\section{Introduction}

Plants produce secondary metabolites as signals to interact with the environment and stresses $[1,2]$. A number of secondary metabolites from seaweeds have been detected to have such valuable bioactivities as antibacterial, anti-viral, anticancer, and antioxidant. In Vietnam, the brown seaweed genus Lobophora belongs to the family Dictyotaceae, found worldwide in tropical to temperate waters and discovered in the coral reef. From the early 1980s to 2017, 49 scientific works have been reported on chemicals and bioactivities of Lobophora genus, in which 40 have been reported with bioactivities. Particularly, most of the studies have been centered on Lobophora variegata, while other species have been still poorly studied and reported [3]. Until now, there have been few studies on lipidomic profile and their bioactivities.
Certain lipid classes have been identified in several algae including polar (Pol), sterol (ST), diacylglycerol (DG), free fatty acids (FFA), triacylglycerol (TG), monoalkyldiacylglycerol (MADG), and hydrocarbons and wax (HW) [4], while neutral lipid classes play various roles such as storing energy or pre-hormones for the body; the polar lipid class has been especially considered for its various bioactivities such as acting as antioxidant and helping in curing cardiovascular diseases and cancer caused by long chain polyunsaturated fatty acids (PUFAs) $[5,6]$. Some high level PUFAs containing C20 fatty acids such as 20:4n6 (AA), 20:5n-3 (EPA), and 20:3n-6 have been identified and evaluated bioactivities in 7 brown seaweed species belonging to the genera Sargassum, Cystoseira, Padina, and Turbinaria. Other PUFAs have been identified as $22: 6 n-3$ (DHA), which is a highly valuable bioactive fatty acid for 
application in medicine and food [7]. In addition to being sources of PUFA, the polar lipids have an important role in structural function as components of cell membranes [8].

In algae, fatty acids are found in the esterified structure such as glycoglycerolipids (GLs), glycerophospholipids (PLs), and betaine lipids [9]. The research on the lipidomics is essential to identify new bioactive compounds and properties based on the polar lipid composition [10]. Moreover, it is also a critical step to the discovery of fostering bio-prospection of lipidic extracts.

Nowadays, mass spectrometry (MS) coupled with liquid chromatography (LC) technique is usually used to determine the detailed structural characterization of lipids and to fully explore lipidomic signature of distinct matrices $[11,12]$ and identify lipidome signature of cultivated seaweeds Ulva lactuca Linnaeus [13], Chondrus crispus Stackhouse [14], and Codium tomentosum Stackhouse [15]. However, there has been no report on Lobophora genus lipidome.

This study aims to analyse and identify the lipid characterisation of species Lobophora sp. first collected at Con Dao, Ba Ria-Vung Tau, Vietnam, by using high-performance liquid chromatography in combination with high-resolution mass spectrometry (HPLC-HRMS). The lipid extracts have also been pre-tested for anti-inflammatory effects by inhibiting the production of NO.

\section{Materials and Methods}

The Lobophora sp. samples were collected in Con Dao, Ba Ria-Vung Tau, Vietnam. Chemicals were obtained from Sigma, Merck, that reached the purification standards for analysis and HPLC grade.

2.1. Total Lipid Extraction. Total lipid was extracted according to method of Bligh and Dyer [16] and Nguyen et al. [17]. Polar lipids were obtained by the silica column. The $200 \mathrm{mg}$ of total lipid was dissolved in chloroform and loaded on the silica column (Phillipsburg, NJ), washed by $44 \mathrm{ml}$ chloroform to remove the pigments and neutral lipid, and then eluted by $120 \mathrm{ml} \mathrm{MeOH} \mathrm{95 \%} \mathrm{to} \mathrm{obtain} \mathrm{polar} \mathrm{lipids.} \mathrm{The} \mathrm{obtained}$ fractions were stored in chloroform at $-5^{\circ} \mathrm{C}$ for analysis.

2.2. Analyses of Lipid Classes. Total lipids were dissolved in $\mathrm{CHCl}_{3}(10 \mathrm{mg} / \mathrm{ml})$ and spotted on the Sorbfil thin plate $(6 \times 6 \mathrm{~cm})$. The $\mathrm{n}$-hexane/diethyl ether/acetic acid $(85 / 15 / 1$, $\mathrm{v} / \mathrm{v} / \mathrm{v})$ solvent was applied to identify neutral lipid layers; then, the $\mathrm{CHCl}_{3} / \mathrm{CH}_{3} \mathrm{OH}(2 / 1, \mathrm{v} / \mathrm{v})$ solvent was applied to identify the polar lipid layer. The TLC was displayed by $10 \%$ $\mathrm{H}_{2} \mathrm{SO}_{4} / \mathrm{CH}_{3} \mathrm{OH}$ reagent at $240^{\circ} \mathrm{C}$ for 10 minutes. The image of thin layer was obtained using Epson Perfection 2400 scanner, Japan, with grayscale mode. The lipid layers on the thin plate and the percentage of the layers were identified by light sensitivity on the Sorbfil TLC Analysis software Video densitometer, Krasnodar, Russia [17, 18].

2.3. Analyses of Fatty Acids. Fatty acid methyl esters (FAMEs) were obtained by incubating total lipid with $2 \%$
$\mathrm{H}_{2} \mathrm{SO}_{4}$ in $\mathrm{CH}_{3} \mathrm{OH}$ at $80^{\circ} \mathrm{C}$ for $2 \mathrm{hrs}$ and then cleaned by TLC in the hexane:diethyl ether, 95/5 (v/v) solvent. FAMEs were analysed on Shimadzu GC-2010 gas chromatograph (Kyoto, Japan) using flame ionization detector (FID) on Capillary Equity 5 (Merck, $\mathrm{L} \times \mathrm{ID} 30 \mathrm{~m} \times 0.25 \mathrm{~mm}, \mathrm{~d} f 0.25 \mu \mathrm{m}$ ). Carrier gas was $\mathrm{He}$ at the speed of $20 \mathrm{ml} / \mathrm{min}$. Temperature program in the oven operating was at $160^{\circ} \mathrm{C}$, raised $2^{\circ} \mathrm{C} / \mathrm{min}$ to $240^{\circ} \mathrm{C}$, and then kept for 20 minutes. Fatty acids were identified by the equivalent retention time value, Equivalent Chain Length, with the standard system of fatty acids C16:0 and $\mathrm{C} 18: 0$. The fatty acid structures were identified by GCMS. The spectra were compared with the NIST library and fatty acid mass spectra archive $[17,18]$.

2.4. Analysis of Molecular Species of Polar Lipids. The molecules of polar lipids were analysed by high-performance liquid chromatography combined high resolution mass spectrometry (HPLC-HRMS) with Shim-Pack diol column (ID $50 \mathrm{~mm} \times 4.6 \mathrm{~mm}$, carrier size $5 \mu \mathrm{m}$, Shimadzu, Kyoto, Japan). The polar lipid classes were separated by HPLC in a solvent A: n-hexane/2-propanol/formic acid/ $\left(\mathrm{C}_{2} \mathrm{H}_{5}\right)_{3} \mathrm{~N}$ and the solvent B: 2-propanol/ $\mathrm{H}_{2} \mathrm{O} /$ formic acid $/\left(\mathrm{C}_{2} \mathrm{H}_{5}\right)_{3} \mathrm{~N}$ [19] and then detected by the high-resolution ion trap over time of mass spectrometry using the LC/MS-IT-TOF (Shimadzu) device, an electronic atomizing ionization source (ESI) device. Flow rate was $0.2 \mathrm{ml} / \mathrm{min}[17,20,21]$.

2.5. Nitric Oxide Production Inhibition Assay. In vitro antiinflammatory activity of lipid samples was elucidated by using nitric oxide (NO) assay as described previously by Mosmann [22] using the Griess reagents (Promega, USA). The RAW 264.7 cells at the concentration of $2 \times 10^{5}$ cells $/ \mathrm{mL}$ were dropped on the 96-well plate and incubated in $37^{\circ} \mathrm{C}$ with $5 \% \mathrm{CO}_{2}$ in $24 \mathrm{~h}$. The culture medium was exchanged to Dulbecco's Modified Eagle's Medium (DMEM) (Life Technologies, USA) with no fetal bovine serum (FBS) for 3 hours. The cells were incubated with the lipid fractions at different concentrations before stimulating the NO production by LPS $(1 \mu \mathrm{g} / \mathrm{mL})$ for 24 hours. Then, the amount of nitrite in the culture medium was measured by Griess reaction at room temperature for 10 minutes. The fresh culture medium was used as a blank, while L-NMMA (Sigma) was tested as the positive sample. The mixtures were quantified spectrophotometrically at $540 \mathrm{~nm}$ using a micro-plate reader (ELx800 Biotekm, USA). The sodium nitrite was used as a standard compound to establish the standard curve. The NO inhibition was calculated following the formula: \% NO inhibition $=100 \%-$ [concentration of $\mathrm{NO}_{\text {sample }} /$ concentration of $\left.\mathrm{NO}_{\mathrm{LPS}}\right] * 100$. IC50s were calculated by TableCurve 2Dv4 software.

The cell viability test was performed parallel with the NO inhibition assay. The 3-(4,5-dimethylthiazol-2-yl)-2,5diphenyltetrazolium bromide (MTT) was used to evaluate cell viability because active cells transform the water-soluble MTT to an insoluble purple formazan. $20 \mu \mathrm{l}$ lipid sample and $180 \mu \mathrm{L}$ of RAW 264.7 cells were put into 96-well plates and then incubated with $5 \% \mathrm{CO}_{2}$ for 72 hours at $37^{\circ} \mathrm{C}$. After adding MTT $(5 \mu \mathrm{g} / \mathrm{mL})$, the mixtures were incubated for 4 
hours and supernatant was removed. The formazan crystals were dissolved in DMSO and measured at $540 \mathrm{~nm}$. The percentage of cell viability was determined by comparing to the control samples.

\section{Results and Discussion}

3.1. Total Lipid. Total lipid content from brown algae Lobophora sp. is $1.06 \pm 0.2 \%$ weight of fresh algae, similar to the brown samples previously studied $[4,23]$. Compositions and contents of total lipid classes of Lobophora sp. are shown in Figure 1 and Table 1. Pol class accounts for the highest level of $26.8 \%$ compared with the detected classes, following by 4 classes of TG, FFA, ST, and HW with the content of $26.0 \%, 25.9 \%, 18.5 \%$, and $2.7 \%$, respectively.

3.2. Fatty Acids Composition. In the lipid composition of Lobophora sp. (Table 2), 23 fatty acids $\mathrm{C} 12-\mathrm{C} 22$ are identified. SAFAs contain 8 fatty acids $12: 0,14: 0,15: 0,16: 0$, $17: 0,18: 0,20: 0$, and $22: 0$, in which $14: 0(9.62 \%)$ and 16 : $0(12.04 \%)$ have high contents. MUFAs group has $16: 1 \mathrm{n}-5$, $16: 1 \mathrm{n}-7,18: 1 \mathrm{n}-7$, and $18: 1 \mathrm{n}-9$, in which $18: 1 \mathrm{n}-9(11.53 \%)$ is typical. PUFAs group has 11 fatty acids including $18: 2 \mathrm{n}-6$, $18: 3 n-3,18: 3 n-6,18: 4 n-3,20: 2 n-6,20: 3 n-6,20: 3 n-9$, $20: 4 n-3,20: 4 n-6$ (AA) $20: 5 n-3$ (EPA), and $22: 6 n-3$ (DHA), in which AA (12.14\%), EPA (11.56\%), and DHA (14.26\%) are significant.

3.3. Molecular Species of Polar Lipids. Three subclasses including phospholipid, glycolipid, and betaine lipid were identified in $26.80 \%$ polar lipid (Pol).

First, the phospholipid subclass contains 4 groups of phosphatidylinositol (PI), phosphatidylcholine (PC), phosphatidylglycerol (PG), and phosphatidic acid (PA). In PI group, 13 molecular forms were found, of which 11 forms had complete formula with one PI isomer form and no alkenyl acyl glycerophosphoinositol (Table 3).

In particular, PI 34:1 is recognized at the highest rate of $44.09 \%$ PI Figure (1S). On the $\mathrm{MS}^{-}$spectrum, the ion $[\mathrm{M}-\mathrm{H}]^{-}$has the strongest signal at $\mathrm{m} / \mathrm{z} 835.5283$ corresponding to ion $\left[\mathrm{C}_{43} \mathrm{H}_{79} \mathrm{O}_{13} \mathrm{P}\right]^{-}$(calculated 836.5415, different 0.00620 ). The $\mathrm{MS}^{2-}$ spectrum shows signals at $\mathrm{m} / \mathrm{z}$ 255.2338 corresponding to the anion of $16: 0$ fatty acids and $\mathrm{m} / \mathrm{z} 281.2471$ to the anion of $18: 1$ fatty acid. Importantly, fragment at $\mathrm{m} / \mathrm{z} 297.0334$ is the considered ion $\left[\mathrm{C}_{43} \mathrm{H}_{79} \mathrm{O}_{13} \mathrm{P}\right]^{-}$that lost the diacyl group which is the characteristic fragmentation to determine the PI molecular forms. Thus, the mass spectrometry data prove that the considered molecule is diacyl glycerophosphoinositol PI 16: $0 / 18: 1$ or PI $34: 1$.

In the PC group, 18 molecular forms have been identified, in which 14 molecular forms are completely identified with one isomer (Table 3). The PC fragmentation has been observed on both $\mathrm{MS}^{-}$and $\mathrm{MS}^{+}$with signals of ions $[\mathrm{M}+\mathrm{H}]^{+},[\mathrm{M}+\mathrm{HCOO}]^{-}$, and $\left[\mathrm{M}-\mathrm{CH}_{3}\right]^{-}$. For example, the PC $30: 0$ with highest content of $19.3 \%$ is recognized with the appearance of $[\mathrm{M}+\mathrm{H}]^{+}$signal at $\mathrm{m} / \mathrm{z} 706.5375$ corresponding to $\left[\mathrm{C}_{38} \mathrm{H}_{77} \mathrm{NO}_{8} \mathrm{P}\right]^{+}$(calculated 705.5309, different
0.00063 ) and two signals at $\mathrm{m} / \mathrm{z} 750.5226$ and $\mathrm{m} / \mathrm{z} 690.5025$ corresponding to ion $[\mathrm{M}+\mathrm{HCOO}]^{-}$and ion $\left[\mathrm{M}-\mathrm{CH}_{3}\right]^{-}$, respectively (Figure $2 \mathrm{~S}$ ). On the $\mathrm{MS}^{2-}$ spectrum, the signal at $\mathrm{m} / \mathrm{z} 690.5088$ is formed by removing a $\mathrm{C}_{2} \mathrm{H}_{4} \mathrm{O}_{2}$ molecule (methyl formate) from the ion at $\mathrm{m} / \mathrm{z} 750.5226$. Besides, two fragments at $\mathrm{m} / \mathrm{z} 227.2042$ corresponding to ion of $14: 0$ fatty acid (calculated 228.2089, different 0.00255 ) and at $\mathrm{m} / \mathrm{z}$ 255.2262 to ion of 16:0 fatty acid (calculated 256.2402, different 0.00675) have resulted. Therefore, PC 30:0 has been identified as diacyl glycerophosphocholine, PC 14:0/ $16: 0$.

In the PG group, 15 molecular forms have been determined with complete formula, in which 6 PGs are detected with isomers (Table 3). PG 34:2 is found at the highest rate of $41.83 \%$. On the $\mathrm{MS}^{-}$, signal of ion $[\mathrm{M}-\mathrm{H}]^{-}$is observed with the strongest intensity at $\mathrm{m} / \mathrm{z} 745.4985$ corresponding to ion $\left[\mathrm{C}_{40} \mathrm{H}_{74} \mathrm{O}_{10} \mathrm{P}\right]^{-}$. The $\mathrm{MS}^{2-}$ of the ion $\left[\mathrm{C}_{40} \mathrm{H}_{74} \mathrm{O}_{10} \mathrm{P}\right]^{-}$ (Figure 3S) contains a signal at $\mathrm{m} / \mathrm{z} 253.2137$ (calculated 254.2246, different 0.0009 ) corresponding to $16: 1$ fatty acid and signal at $\mathrm{m} / \mathrm{z} 281.2485$ (calculated 282.2559, different 0.0026 ) corresponding to $18: 1$ fatty acid. Besides, there have been 3 signals including at $\mathrm{m} / \mathrm{z} 417.2395 ; 491.2738 ; 509.2838$ corresponding to molecules which have lost glycerol and acyl groups of 16:1 fatty acid anion; a 16:1 fatty acid; a ketene group of $16: 1$ fatty acid anion, respectively. In addition, the $\mathrm{MS}^{2-}$ (Figure 3S) shows a lower intensity signal at $\mathrm{m} / \mathrm{z} 279.2349$ (calculated 280.2402, different 0.00195) corresponding to the carboxylate anion of $18: 2$ fatty acid. This indicates that there is an isomer of PG $34: 2$ with composition containing $18: 2$ and $16: 0$ fatty acids. The above data demonstrate that the molecular ion value $\mathrm{m} / \mathrm{z} 745.4985$ presents two isomers including PG $16: 0 / 18: 2$ and PG $16: 1 /$ $18: 1$, in which PG $16: 1 / 18: 1$ is the major content.

With the PA molecular forms, only one PA 40:8 is identified (Table 3). On the $\mathrm{MS}^{-}$spectrum of PA, ion $[\mathrm{M}-\mathrm{H}]^{-}$ is observed with the strongest signal at $\mathrm{m} / \mathrm{z} 743.4609$ (calculated 744.4730, different $0.00483,11$ double bonds) corresponding to ion $\left[\mathrm{C}_{43} \mathrm{H}_{68} \mathrm{O}_{8} \mathrm{P}\right]^{-}$(Figure $4 \mathrm{~S}$ ). On the $\mathrm{MS}^{2-}$, fragments have been found with the signals at $\mathrm{m} / \mathrm{z} 457.2260$ and $\mathrm{m} / \mathrm{z} 439.2215$ corresponding to the considered PA fragment which has lost a dehydrated molecule of $20: 4$ and a $20: 4$ fatty acids, respectively. Additionally, the signal at $\mathrm{m} /$ z 303.2243 is the acyl carboxylate ion of $20: 4$ fatty acid. With the above data, PA 40:8 is identified as diacyl glycerophosphatic acid PA $20: 4 / 20: 4$.

Second, in the glycolipid subclass, 3 groups have been identified including monogalactosyldiacylglycerol (MGDG), digalactosyldiacylglycerol (DGDG), and sulfoquinovosyldiacylglycerol (SQDG). In the MGDG group, 21 molecular forms are identified, in which 8 have been completely identified (Table 4).

For example, with the highest ratio of $13.92 \%$, MGDG $38: 9$ has formed molecular ions (Figure 5S). On the $\mathrm{MS}^{-}$, the ion $[\mathrm{M}+\mathrm{HCOO}]^{-}$signal at $\mathrm{m} / \mathrm{z} 841.5021$ corresponds to ion $\left[\mathrm{C}_{48} \mathrm{H}_{73} \mathrm{O}_{12}\right]^{-}$and the ion $[\mathrm{M}-\mathrm{H}]^{-}$signal at $\mathrm{m} / \mathrm{z}$ 795.5046 corresponds to ion $\left[\mathrm{C}_{47} \mathrm{H}_{71} \mathrm{O}_{10}\right]^{-}$. On the $\mathrm{MS}^{+}$, the ion $[\mathrm{M}+\mathrm{Na}]^{+}$has the strongest signal at $\mathrm{m} / \mathrm{z} 819.5023$ corresponding to $\left[\mathrm{C}_{47} \mathrm{H}_{72} \mathrm{O}_{10} \mathrm{Na}\right]^{+}$. On the $\mathrm{MS}^{2+}$ of $\left[\mathrm{C}_{47} \mathrm{H}_{72} \mathrm{O}_{10} \mathrm{Na}\right]^{+}$ion, it has been simultaneously observed 


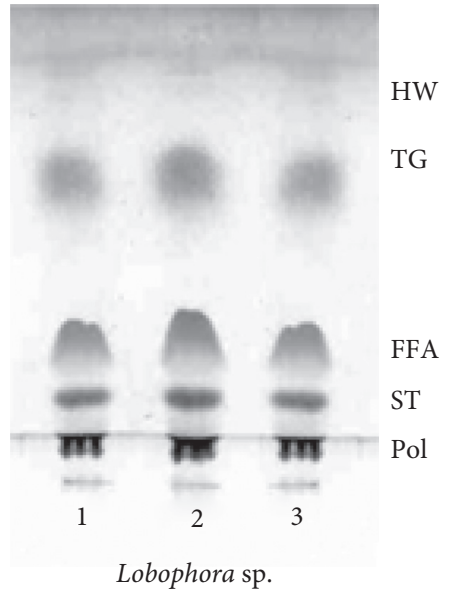

FIGURE 1: TLC for determination of total lipid.

TABle 1: Five main classes (\% of total lipid) of Lobophora sp.

\begin{tabular}{lc}
\hline Lipid class & Content (\%) \\
\hline Hydrocarbon and wax (HW) & $2.7 \pm 0.1$ \\
Triacylglycerol (TG) & $26.1 \pm 0.3$ \\
Free fatty acids (FFA) & $25.9 \pm 0.6$ \\
Sterols (ST) & $18.5 \pm 0.4$ \\
Polar lipid (Pol) & $26.8 \pm 0.5$ \\
\hline
\end{tabular}

TABLE 2: Fatty acid profile (\% of total) is identified by GC through the retention time, presented with relative abundance (\%) through the area of peaks.

\begin{tabular}{lccc}
\hline FA & R. t (min) & Area & Content $(\%)$ \\
\hline$\sum$ SAFAs & & 415367.3 & $\mathbf{2 2 . 8 0}$ \\
$12: 0$ & 10.378 & 2540.9 & 0.14 \\
$14: 0$ & 11.79 & 175274.9 & 9.62 \\
$15: 0$ & 15.045 & 5711.9 & 0.31 \\
$16: 0$ & 18.725 & 219431.0 & 12.04 \\
$17: 0$ & 21.663 & 3011.5 & 0.17 \\
$18: 0$ & 26.624 & 3236.6 & 0.18 \\
$20: 0$ & 39.466 & 1788.1 & 0.10 \\
$22: 0$ & 53.211 & 4372.4 & 0.24 \\
\hline$\sum$ MUFAs & & 275411.2 & $\mathbf{1 5 . 1 2}$ \\
$16: 1 \mathrm{n}-5$ & 18.541 & 20962.8 & 1.15 \\
$16: 1 \mathrm{n}-7$ & 17.931 & 36794.7 & 2.02 \\
$18: 1 \mathrm{n}-7$ & 25.799 & 10957.1 & 0.60 \\
$18: 1 \mathrm{n}-9$ & 25.600 & 206696.6 & 11.35 \\
\hline$\sum$ PUFAs & & 1092787.0 & $\mathbf{5 9 . 9 8}$ \\
$18: 2 \mathrm{n}-6$ & 24.949 & 83066.0 & 4.56 \\
$18: 3 \mathrm{n}-3$ & 25.334 & 122483.9 & 6.72 \\
$18: 3 \mathrm{n}-6$ & 24.687 & 95003.7 & 5.21 \\
$18: 4 \mathrm{n}-3$ & 24.826 & 21806.6 & 1.20 \\
$20: 2 \mathrm{n}-6$ & 33.406 & 6677.5 & 0.37 \\
$20: 3 \mathrm{n}-6$ & 32.921 & 20389.4 & 1.12 \\
$20: 3 \mathrm{n}-9$ & 32.655 & 44276.1 & 2.43 \\
$20: 4 \mathrm{n}-3$ & 32.444 & 7549.2 & 0.41 \\
$20: 4 \mathrm{n}-6$ (AA) & 31.944 & 221189.9 & 12.14 \\
$20: 5 \mathrm{n}-3$ (EPA) & 32.212 & 210603.5 & 11.56 \\
$22: 6 \mathrm{n}-3$ (DHA) & 43.138 & 259741.5 & 14.26 \\
\hline Other & & 38246.8 & 2.10 \\
\hline
\end{tabular}

${ }^{a}$ Hexadecanal, octadecenal, and fatty aldehyde are dimethylacetal derivatives determined by GC; SAFAs: saturated fatty acids; MUFAs: monounsaturated fatty acids; PUFAs: polyunsaturated fatty acids.
TABle 3: Molecular species of phospholipid including PI, PC, PG, and PA groups identified by HPLC-HRMS as negative $[\mathrm{M}-\mathrm{H}]^{-}$ ion and positive $[\mathrm{M}+\mathrm{H}]^{+}$ion, and identification as phospholipid and fatty acyl composition confirmed by the analysis of the LC-MS/ MS spectra of each ion.

\begin{tabular}{|c|c|c|c|}
\hline$[\mathrm{M}-\mathrm{H}]^{-} \mathrm{m} / \mathrm{z}$ & PI & Fatty acid chains & $\%$ in $\mathrm{PI}$ \\
\hline 781.4797 & $30: 0$ & $14: 0 / 16: 0$ & 1.81 \\
\hline \multirow[t]{2}{*}{805.483} & $32: 2$ & $14: 0 / 18: 2$ & 0.82 \\
\hline & $32: 1$ & $14: 0 / 18: 1$ & 6.27 \\
\hline 807.4966 & & $16: 0 / 16: 1$ & \\
\hline 809.5144 & $32: 0$ & $16: 0 / 16: 0$ & 2.39 \\
\hline 821.515 & $33: 1$ & & 0.97 \\
\hline 829.4807 & $34: 4$ & & 1.67 \\
\hline 831.4957 & $34: 3$ & $16: 0 / 18: 3$ & 2.60 \\
\hline 833.512 & $34: 2$ & $16: 0 / 18: 2$ & 8.77 \\
\hline 835.5283 & $34: 1$ & $16: 0 / 18: 1$ & 44.09 \\
\hline 845.5116 & $35: 3$ & & 1.63 \\
\hline 855.4971 & $36: 5$ & $16: 0 / 20: 5$ & 1.40 \\
\hline 857.5103 & $36: 4$ & $16: 0 / 20: 4$ & 15.43 \\
\hline 859.5269 & $36: 3$ & $16: 0 / 20: 3$ & 12.15 \\
\hline$[\mathrm{M}+\mathrm{H}]^{+} \mathrm{m} / \mathrm{z}$ & $\mathrm{PC}$ & Fatty acid chains & $\%$ in $\mathrm{PC}$ \\
\hline 678.5056 & $28: 0$ & $14: 0 / 14: 0$ & 15.33 \\
\hline 706.5375 & $30: 0$ & $14: 0 / 16: 0$ & 19.30 \\
\hline \multirow[t]{2}{*}{732.5494} & $32: 1$ & $14: 0 / 18: 1$ & 6.44 \\
\hline & & $16: 0 / 16: 1$ & \\
\hline 734.5677 & $32: 0$ & $16: 0 / 16: 0$ & 4.07 \\
\hline 754.5364 & $34: 4$ & $14: 0 / 20: 4$ & 7.81 \\
\hline 756.5515 & $34: 3$ & $14: 0 / 20: 3$ & 3.25 \\
\hline 758.5678 & $34: 2$ & $16: 0 / 18: 2$ & 5.84 \\
\hline 760.5819 & $34: 1$ & $16: 0 / 18: 1$ & 7.00 \\
\hline 762.6003 & $34: 0$ & $14: 0 / 20: 0$ & 1.06 \\
\hline 768.5880 & $36: 3$ & & 0.20 \\
\hline 780.5566 & $36: 5$ & & 1.69 \\
\hline 782.5719 & $36: 4$ & $16: 0 / 20: 4$ & 10.64 \\
\hline 784.5839 & $36: 3$ & $16: 0 / 20: 3$ & 5.54 \\
\hline 786.5970 & $36: 2$ & $16: 0 / 20: 2$ & 2.66 \\
\hline 806.5627 & $38: 4$ & & 2.21 \\
\hline 808.5837 & $38: 5$ & $18: 1 / 20: 4$ & 5.31 \\
\hline 810.5933 & $38: 2$ & & 1.47 \\
\hline 838.6291 & $40: 4$ & & 0.18 \\
\hline$[\mathrm{M}-\mathrm{H}]^{-} \mathrm{m} / \mathrm{z}$ & PG & Fatty acid chains of PG & $\%$ in $\mathrm{PG}$ \\
\hline 691.4524 & $30: 1$ & $14: 0 / 16: 1$ & 0.31 \\
\hline 693.469 & $30: 0$ & $14: 0 / 16: 0$ & 0.74 \\
\hline \multirow[t]{2}{*}{717.4698} & $32: 2$ & $16: 1 / 16: 1$ & 0.85 \\
\hline & & $14: 1 / 18: 1$ & \\
\hline 719.4851 & $32: 1$ & $16: 0 / 16: 1$ & 4.94 \\
\hline 721.5006 & $32: 0$ & $16: 0 / 16: 0$ & 1.07 \\
\hline \multirow[t]{2}{*}{741.4682} & $34: 4$ & $16: 1 / 18: 3$ & 1.38 \\
\hline & & $16: 1 / 18: 2$ & 11.07 \\
\hline \multirow[t]{2}{*}{743.4833} & $34: 3$ & $16: 0 / 18: 3$ & \\
\hline & & $16: 2 / 18: 1$ & \\
\hline \multirow[t]{2}{*}{745.4985} & $34: 2$ & $16: 1 / 18: 1$ & 41.83 \\
\hline & & $16: 0 / 18: 2$ & \\
\hline \multirow[t]{2}{*}{747.5158} & $34: 1$ & $16: 0 / 18: 1$ & 14.27 \\
\hline & & $18: 0 / 16: 1$ & \\
\hline 765.4675 & $36: 6$ & $16: 1 / 20: 5$ & 4.68 \\
\hline \multirow[t]{2}{*}{767.482} & $36: 5$ & $16: 0 / 20: 5$ & 11.22 \\
\hline & & $16: 1 / 20: 4$ & \\
\hline \multirow[t]{2}{*}{769.4938} & $36: 4$ & $20: 4 / 16: 0$ & 1.72 \\
\hline & & $20: 3 / 16: 1$ & \\
\hline 771.5138 & $36: 3$ & $16: 0 / 20: 3$ & 1.64 \\
\hline 773.531 & $36: 2$ & $18: 1 / 18: 1$ & 3.83 \\
\hline 793.4985 & $35: 6$ & $18: 1 / 20: 5$ & 0.45 \\
\hline$[\mathrm{M}-\mathrm{H}]^{-} \mathrm{m} / \mathrm{z}$ & $\mathrm{PA}$ & Fatty acid chains & $\%$ in $\mathrm{PA}$ \\
\hline 743.4609 & $40: 8$ & $20: 4 / 20: 4$ & 100 \\
\hline
\end{tabular}


TABLE 4: Molecular species of glycolipid including MGDG, DGDG, and SQDG groups identified by HPLC-HRMS as negative $[\mathrm{M}-\mathrm{H}]^{-}$ ions and positive $[\mathrm{M}+\mathrm{Na}]^{+}$ion, and identification as phospholipid and fatty acyl composition confirmed by the analysis of the LC-MS/ MS spectra of each ion.

\begin{tabular}{|c|c|c|c|}
\hline$[\mathrm{M}+\mathrm{Na}]^{+} \mathrm{m} / \mathrm{z}$ & MGDG & Fatty acid chains & $\%$ in $\mathrm{MGDG}$ \\
\hline 725.5160 & $30: 0$ & \multirow{7}{*}{$14: 0 / 18: 1$} & 1.96 \\
\hline 747.3232 & $32: 3$ & & 1.00 \\
\hline 749.5268 & $32: 2$ & & 1.75 \\
\hline 751.5297 & $32: 1$ & & 9.39 \\
\hline 769.4902 & $34: 6$ & & 1.23 \\
\hline 771.4933 & $34: 5$ & & 2.07 \\
\hline 775.5024 & $34: 3$ & & 1.81 \\
\hline 777.5466 & $34: 2$ & \multirow{3}{*}{$16: 0 / 18: 1$} & 2.25 \\
\hline 779.5654 & $34: 1$ & & 13.54 \\
\hline 793.4879 & $36: 8$ & & 0.87 \\
\hline 795.4995 & $36: 7$ & \multirow{5}{*}{$16: 0 / 20: 5$} & 1.56 \\
\hline 797.5172 & $36: 6$ & & 5.37 \\
\hline 799.5326 & $36: 5$ & & 7.85 \\
\hline 801.5539 & $36: 4$ & & 3.69 \\
\hline 803.5563 & $36: 3$ & & 1.71 \\
\hline 819.5023 & $38: 9$ & $18: 4 / 20: 5$ & 13.92 \\
\hline 821.5147 & $38: 8$ & $18: 3 / 20: 5$ & 13.73 \\
\hline 823.5270 & $38: 7$ & $18: 3 / 20: 4$ & 9.12 \\
\hline 825.5550 & $38: 6$ & $18: 1 / 20: 5$ & 3.64 \\
\hline 849.3232 & $40: 8$ & \multirow[t]{2}{*}{$20: 4 / 20: 4$} & 2.24 \\
\hline 851.5628 & $40: 7$ & & 1.30 \\
\hline$[\mathrm{M}+\mathrm{Na}]^{+} \mathrm{m} / \mathrm{z}$ & DGDG & Fatty acid chains & $\%$ in $\mathrm{DGDG}$ \\
\hline 941.6144 & $34: 1$ & $16: 0 / 18: 1$ & 100 \\
\hline$[\mathrm{M}-\mathrm{H}]^{-} \mathrm{m} / \mathrm{z}$ & SQDG & Fatty acid chains & $\%$ in SQDG \\
\hline \multirow{2}{*}{737.4474} & \multirow{2}{*}{$28: 0$} & $14: 0 / 14: 0$ & \multirow{2}{*}{1.63} \\
\hline & & $12: 0 / 16: 0$ & \\
\hline 751.4631 & $29: 0$ & $14: 0 / 15: 0$ & 0.44 \\
\hline 763.4619 & $30: 1$ & $14: 0 / 16: 1$ & 0.74 \\
\hline 765.4771 & $30: 0$ & $14: 0 / 16: 0$ & 21.78 \\
\hline 779.4909 & $31: 0$ & $15: 0 / 16: 0$ & 0.63 \\
\hline 789.4757 & $32: 2$ & $14: 0 / 18: 2$ & 0.71 \\
\hline \multirow[t]{2}{*}{791.4931} & \multirow[t]{2}{*}{$32: 1$} & $14: 0 / 18: 1$ & \multirow[t]{2}{*}{9.70} \\
\hline & & $16: 0 / 16: 1$ & \\
\hline 793.5107 & $32: 0$ & $16: 0 / 16: 0$ & 13.68 \\
\hline \multirow[t]{2}{*}{815.4871} & \multirow[t]{2}{*}{$34: 3$} & $16: 0 / 18: 3$ & \multirow[t]{2}{*}{0.73} \\
\hline & & $14: 0 / 20: 3$ & \\
\hline \multirow[t]{2}{*}{817.5065} & \multirow[t]{2}{*}{$34: 2$} & $16: 0 / 18: 2$ & \multirow[t]{2}{*}{3.21} \\
\hline & & $16: 1 / 18: 1$ & \\
\hline 819.5162 & $34: 1$ & $16: 0 / 18: 1$ & 21.22 \\
\hline \multirow[t]{2}{*}{821.5376} & \multirow[t]{2}{*}{$34: 0$} & $16: 0 / 18: 0$ & \multirow[t]{2}{*}{5.83} \\
\hline & & $14: 0 / 20: 0$ & \\
\hline 833.5376 & $35: 1$ & $17: 0 / 18: 1$ & 0.77 \\
\hline & & $16: 0 / 19: 1$ & \\
\hline 835.5563 & $35: 0$ & $16: 0 / 19: 0$ & 0.10 \\
\hline 837.4736 & $36: 6$ & $16: 0 / 20: 6$ & 1.58 \\
\hline 839.4834 & $36: 5$ & $16: 0 / 20: 5$ & 2.30 \\
\hline 841.4987 & $36: 4$ & $16: 0 / 20: 4$ & 2.10 \\
\hline 843.5163 & $36: 3$ & $16: 0 / 20: 3$ & 0.45 \\
\hline 845.5369 & $36: 2$ & $16: 0 / 20: 2$ & 1.79 \\
\hline & & $18: 1 / 18: 1$ & \\
\hline 847.5562 & $36: 1$ & $18: 0 / 18: 1$ & 0.61 \\
\hline & & $16: 0 / 20: 1$ & \\
\hline 849.5704 & $36: 0$ & $16: 0 / 20: 0$ & 1.48 \\
\hline & & $18: 0 / 18: 0$ & \\
\hline 877.6017 & $38: 0$ & $16: 0 / 22: 0$ & 0.71 \\
\hline
\end{tabular}

TABle 4: Continued.

\begin{tabular}{lccc}
\hline$[\mathrm{M}+\mathrm{Na}]^{+} \mathrm{m} / \mathrm{z}$ & MGDG & Fatty acid chains & \% in MGDG \\
\hline & & $14: 0 / 24: 0$ \\
903.6170 & $40: 1$ & $16: 0 / 24: 1$ & 0.17 \\
905.6325 & $40: 0$ & $16: 0 / 24: 0$ & 4.85 \\
931.6486 & $42: 1$ & $16: 0 / 26: 1$ & 0.71 \\
933.6654 & $42: 0$ & $16: 0 / 26: 0$ & 2.08 \\
\hline
\end{tabular}

with two signals at $\mathrm{m} / \mathrm{z} 543.2932$ and 517.2795 corresponding to the considered MGDG molecule lost fragments of $\mathrm{m} / \mathrm{z} 276.2091\left[\mathrm{C}_{18} \mathrm{H}_{27} \mathrm{O}_{2}\right]^{-}$(18:4 fatty acid) and $\mathrm{m} / \mathrm{z}$ $302.2228\left[\mathrm{C}_{20} \mathrm{H}_{29} \mathrm{O}_{2}\right]^{-}(20: 5$ fatty acid $)$, respectively. The obtained data show that the MGDG $38: 9$ is $18: 4 / 20: 5$.

In the DGDG group, only one DGDG form has been found as DGDG 34:1 which is described in Table 4 and Figure 6S. On the $\mathrm{MS}^{+}$spectrum, the ion $[\mathrm{M}+\mathrm{Na}]^{+}$has the signal at $\mathrm{m} / \mathrm{z} 941.6144$ corresponding to ion $\left[\mathrm{C}_{49} \mathrm{H}_{90} \mathrm{O}_{15} \mathrm{Na}\right]^{+}$. On the $\mathrm{MS}^{-}$spectrum, the ion $[\mathrm{M}-\mathrm{H}]^{-}$has the strongest signal at $\mathrm{m} / \mathrm{z} 917.6080$ corresponding to ion $\left[\mathrm{C}_{49} \mathrm{H}_{89} \mathrm{O}_{15}\right]^{-}$ (calculated 917.6116, different 0.00580). On the $\mathrm{MS}^{2-}$ spectrum of ion $\left[\mathrm{C}_{49} \mathrm{H}_{89} \mathrm{O}_{15}\right]^{-}$, there have been simultaneously signals at $\mathrm{m} / \mathrm{z} 661.3696$ and $\mathrm{m} / \mathrm{z} 635.3551$ corresponding to the considered DGDG molecule that has lost neutral fragments including 16:0 fatty acid ( $\mathrm{m} / \mathrm{z} 256.2420)$ and $18: 1$ fatty acid $(\mathrm{m} / \mathrm{z} 282.2565)$. The above data indicate that the molecular form is DGDG $16: 0 / 18: 1$.

In the SQDG group, 26 complete molecular forms have been identified with 10 isomers (Table 4). A molecule with the highest content of $21.78 \%$ is identified as SQDG $34: 1$. On the $\mathrm{MS}^{-}$spectrum, the ion $[\mathrm{M}-\mathrm{H}]^{-}$of SQDG $34: 1$ is observed with the strongest signal at $\mathrm{m} / \mathrm{z} 819.5162$ corresponding to ion $\left[\mathrm{C}_{43} \mathrm{H}_{79} \mathrm{O}_{12} \mathrm{~S}\right]^{-}$(Figure 7S). On the $\mathrm{MS}^{2-}$ spectrum, the signal at $\mathrm{m} / \mathrm{z} 581.2991$ is formed when the ion at $\mathrm{m} / \mathrm{z} 819.5162$ eliminates a dehydrated molecule of fatty acid 16:0. The signal at $\mathrm{m} / \mathrm{z} 563.2831$ has also been observed in the $\mathrm{MS}^{2-}$ spectrum simultaneously. This signal appears when the ion $\left[\mathrm{C}_{43} \mathrm{H}_{79} \mathrm{O}_{12} \mathrm{~S}\right]^{-}$loses fatty acid 16:0. In addition, the appearance of signal $\mathrm{m} / \mathrm{z} 281.2489$ (calculated 282.2559, different 0.00030 ) corresponds to anion of $18: 1$ fatty acid. Thus, data show that the obtained SQDG $34: 1$ is $16: 0 / 18: 1$.

In addition to phospholipid and glycolipid classes, betaine lipid subclass includes 2 groups of DGTA and DGTS. In the DGTA group, 37 molecular forms have been identified, in which 29 forms are completely identified with 8 isomers (Table 5). Among those, DGTA $34: 1$ is the highest content of $10.20 \%$. On the $\mathrm{MS}^{+}$spectrum, the ion $[\mathrm{M}+\mathrm{H}]^{+}$ has the strongest signal at $\mathrm{m} / \mathrm{z} 738.6298$ corresponding to ion $\left[\mathrm{C}_{44} \mathrm{H}_{84} \mathrm{NO}_{7}\right]^{+}$. On the $\mathrm{MS}^{2+}$ spectrum, there have been simultaneously two signals at $\mathrm{m} / \mathrm{z} 500.3955$ and 482.3784 corresponding to the ion $\left[\mathrm{C}_{44} \mathrm{H}_{84} \mathrm{NO}_{7}\right]^{+}$that has lost a dehydrated molecule of fatty acid 16:0 and a neutral fragment of 16:0 fatty acid. Besides, the ion $\left[\mathrm{C}_{44} \mathrm{H}_{84} \mathrm{NO}_{7}\right]^{+}$ has lost a dehydrated molecule of $18: 1$ fatty acid and formed a signal at $\mathrm{m} / \mathrm{z} 474.3783$. This ion eliminates water molecule and shows signal at $\mathrm{m} / \mathrm{z} 456.3806$. In addition, the signal at $\mathrm{m} / \mathrm{z} 236.1485$ is semimolecule ion of DGTA that lost diacyl groups, which is a very important signal in determining the 
TABLE 5: Molecular species of betaine lipid including DGTA and DGTS groups identified by HPLC-HRMS as positive $[\mathrm{M}+\mathrm{H}]^{+}$ion, and identification as phospholipid and fatty acyl composition confirmed by the analysis of the LC-MS/MS spectra of each ion.

\begin{tabular}{|c|c|c|c|}
\hline$[\mathrm{M}+\mathrm{H}]^{+} \mathrm{m} / z$ & DGTA & Fatty acid chains & $\%$ in DGTA \\
\hline 656.5454 & $28: 0$ & $14: 0 / 14: 0$ & 1.73 \\
\hline 682.5651 & $30: 1$ & $14: 0 / 16: 1$ & 3.06 \\
\hline 684.5841 & $30: 0$ & & 1.33 \\
\hline 706.5612 & $32: 3$ & $14: 0 / 18: 3$ & 2.60 \\
\hline 708.5813 & $32: 2$ & $14: 0 / 18: 2$ & 9.89 \\
\hline 710.5983 & $32: 1$ & $\begin{array}{l}14: 0 / 18: 1 \\
16: 0 / 16: 1\end{array}$ & 10.12 \\
\hline 712.6104 & $32: 0$ & & 0.06 \\
\hline 724.617 & $33: 1$ & & 0.54 \\
\hline 728.5457 & $34: 6$ & $14: 0 / 20: 6$ & 0.57 \\
\hline 730.5541 & $34: 5$ & $14: 0 / 20: 5$ & 3.67 \\
\hline 732.5674 & $34: 4$ & $14: 0 / 20: 4$ & 9.02 \\
\hline 734.5828 & $34: 3$ & $\begin{array}{l}14: 0 / 20: 3 \\
16: 0 / 18: 3\end{array}$ & 5.04 \\
\hline 736.6094 & $34: 2$ & $\begin{array}{l}16: 0 / 18: 2 \\
14: 0 / 20: 2\end{array}$ & 7.47 \\
\hline 738.6298 & $34: 1$ & $16: 0 / 18: 1$ & 10.20 \\
\hline 752.6434 & $35: 1$ & $\begin{array}{l}16: 0 / 19: 1 \\
17: 0 / 18: 1\end{array}$ & 0.47 \\
\hline 756.5699 & $36: 6$ & $16: 0 / 20: 6$ & 0.88 \\
\hline 758.5821 & $36: 5$ & $16: 0 / 20: 5$ & 1.83 \\
\hline 760.6001 & $36: 4$ & $16: 0 / 20: 4$ & 6.17 \\
\hline 762.6193 & $36: 3$ & $\begin{array}{l}16: 0 / 20: 3 \\
18: 1 / 18: 2\end{array}$ & 7.39 \\
\hline 764.6342 & $36: 2$ & & 4.33 \\
\hline 766.6548 & $36: 1$ & $\begin{array}{l}18: 0 / 18: 1 \\
16: 0 / 20: 1\end{array}$ & 1.40 \\
\hline 782.5867 & $38: 7$ & & 0.37 \\
\hline 784.6011 & $38: 6$ & $18: 2 / 20: 4$ & 1.63 \\
\hline 786.6138 & $38: 5$ & $18: 1 / 20: 4$ & 2.13 \\
\hline 788.6306 & $38: 4$ & $18: 1 / 20: 3$ & 1.03 \\
\hline 790.6501 & $38: 3$ & $\begin{array}{l}18: 0 / 20: 3 \\
18: 1 / 20: 2\end{array}$ & 0.68 \\
\hline 792.6621 & $38: 2$ & & 0.27 \\
\hline 794.6813 & $38: 1$ & $18: 1 / 20: 0$ & 0.20 \\
\hline 804.5707 & $40: 10$ & $20: 4 / 20: 6$ & 0.49 \\
\hline 806.5888 & $40: 9$ & $20: 4 / 20: 5$ & 0.52 \\
\hline 808.6031 & $40: 8$ & $20: 4 / 20: 4$ & 1.45 \\
\hline 810.617 & $40: 7$ & $20: 3 / 20: 4$ & 1.72 \\
\hline 812.6332 & $40: 6$ & $\begin{array}{l}20: 2 / 20: 4 \\
20: 3 / 20: 3\end{array}$ & 0.98 \\
\hline 814.6478 & $40: 5$ & $\begin{array}{l}20: 1 / 20: 4 \\
20: 2 / 20: 3\end{array}$ & 0.44 \\
\hline 848.6329 & $43: 9$ & & 0.32 \\
\hline$[\mathrm{M}+\mathrm{H}]^{+} \mathrm{m} / \mathrm{z}$ & DGTS & Fatty acid chains & $\%$ in DGTS \\
\hline 656.5455 & $28: 0$ & $14: 0 / 14: 0$ & 19.21 \\
\hline 682.5609 & $30: 1$ & $\begin{array}{l}14: 0 / 16: 1 \\
14: 1 / 16: 0\end{array}$ & 1.34 \\
\hline 684.5776 & $30: 0$ & $14: 0 / 16: 0$ & 9.71 \\
\hline 706.5524 & $32: 3$ & $14: 0 / 18: 3$ & 3.25 \\
\hline 708.5767 & $32: 2$ & $\begin{array}{l}14: 0 / 18: 2 \\
16: 0 / 16: 2\end{array}$ & 4.78 \\
\hline 710.5923 & $32: 1$ & $\begin{array}{l}14: 0 / 18: 1 \\
16: 0 / 16: 1\end{array}$ & 12.14 \\
\hline 712.6104 & $32: 0$ & $\begin{array}{l}16: 0 / 16: 0 \\
14: 0 / 18: 0\end{array}$ & 5.03 \\
\hline 724.6059 & $33: 1$ & & 1.80 \\
\hline
\end{tabular}

TABle 5: Continued.

\begin{tabular}{lccc}
\hline$[\mathrm{M}+\mathrm{H}]^{+} \mathrm{m} / z$ & DGTA & Fatty acid chains & \% in DGTA \\
\hline 730.5586 & $34: 5$ & & 1.82 \\
732.5736 & $34: 4$ & $16: 0 / 18: 4$ & 5.20 \\
& & $14: 0 / 20: 4$ & \\
734.5875 & $34: 3$ & $16: 1 / 18: 3$ & 5.15 \\
& & $14: 0 / 18: 3$ & \\
736.6072 & $34: 2$ & $16: 1 / 18: 2$ & \\
& & $16: 0 / 18: 2$ & 7.29 \\
738.6226 & $34: 1$ & $16: 0 / 18: 1$ & 12.20 \\
752.634 & $35: 1$ & & 1.30 \\
756.576 & $36: 6$ & & 1.55 \\
758.5891 & $36: 5$ & & 1.07 \\
760.6036 & $36: 4$ & $16: 0 / 20: 4$ & 3.39 \\
762.6204 & $36: 3$ & $18: 1 / 18: 3$ & \\
764.6344 & $36: 2$ & $18: 1 / 18: 1$ & 2.62 \\
& & $16: 0 / 20: 2$ & 4.76 \\
766.6518 & $36: 1$ & $18: 0 / 18: 2$ & \\
784.6068 & $38: 6$ & & 1.24 \\
786.6186 & $38: 5$ & $18: 1 / 20: 4$ & 1.54 \\
788.6389 & $38: 4$ & $16: 0 / 22: 5$ & 0.63 \\
\hline
\end{tabular}

molecular forms of DGTA (Figure 8S). Thus, the above data prove that the considered molecule is DGTA 16:0/18:1.

There are 23 molecular forms with 13 isomers identified in DGTS subclass (Table 5). The DGTS molecular form at the highest ratio of $19.21 \%$ is DGTS $28: 0$. On the $\mathrm{MS}^{+}$spectrum, the ion $[\mathrm{M}+\mathrm{H}]^{+}$has the strongest signal at $\mathrm{m} / \mathrm{z} 656.5459$ corresponding to ion $\left[\mathrm{C}_{38} \mathrm{H}_{74} \mathrm{NO}_{7}\right]^{+}$(Figure 9S). On the $\mathrm{MS}^{2+}$ spectrum, signal at $\mathrm{m} / \mathrm{z} 446.3490$ appears because the ion $\left[\mathrm{C}_{38} \mathrm{H}_{74} \mathrm{NO}_{7}\right]^{+}$loses a dehydrated molecule of acid $14: 0$. Signal at $\mathrm{m} / \mathrm{z} 428.3395$ has also been observed in the $\mathrm{MS}^{2+}$ spectrum. This signal corresponds to the ion $\left[\mathrm{C}_{38} \mathrm{H}_{74} \mathrm{NO}_{7}\right]^{+}$ that loses a neutral fragment of $14: 0$ fatty acid. In addition, the appearance of signal at $\mathrm{m} / \mathrm{z} 236.1517$ is important for detection of the molecular form of DGTS. It is similar to the determination of DGTA. Thus, the above data prove that the considered molecule is DGTS 14:0/14:0.

3.4. Anti-Inflammatory Activity. Three lipid fractions yield the inhibitory effects on NO production in RAW 264.7 cells. The $\mathrm{IC}_{50}$ values have been evaluated from 52.10 to $66.21 \mu \mathrm{g} /$ $\mathrm{mL}$ (Table 6). The unpolar lipid fraction (UPol) has a strongest activity $\mathrm{IC}_{50}$ of $52.10 \pm 4.43$, following by total lipid and polar lipid samples with $\mathrm{IC}_{50}$ of $61.09 \pm 6.06$ and $66.21 \pm 6.24 \mu \mathrm{g} / \mathrm{mL}$, respectively. All fractions do not exhibit the cytotoxicity with the cell viability from 96.06 to $100 \%$ at the concentration of $100 \mu \mathrm{g} / \mathrm{mL}$, while the control sample shows the cell viability percentage of $89.90 \%$ with the same concentration (Table 6).

\section{Discussion}

In the total lipid of Lobophora sp., 5 classes including Pol, ST, FFA, TG, and HW have been found, while other brown algae 
TABLE 6: The NO inhibition and the cell viability of lipid samples.

\begin{tabular}{|c|c|c|c|c|c|c|c|c|}
\hline \multirow{2}{*}{$\begin{array}{l}\text { Concentration } \\
(\mu \mathrm{g} / \mathrm{mL})\end{array}$} & \multicolumn{2}{|c|}{ L-NMMA } & \multicolumn{2}{|c|}{ Pol } & \multicolumn{2}{|c|}{ UPol } & \multicolumn{2}{|c|}{$\mathrm{TL}$} \\
\hline & Cell viability & NO inhibition & Cell viability & NO inhibition & Cell viability & NO inhibition & Cell viability & NO inhibition \\
\hline 100 & 89.90 & 99.74 & 97.29 & 69.07 & 98.24 & 84.75 & 96.06 & 80.93 \\
\hline 20 & 97.54 & 72.44 & 98.00 & 18.47 & 102.00 & 20.76 & 99.47 & 15.25 \\
\hline 4 & NA & 28.18 & NA & 4.66 & NA & 7.20 & NA & 5.86 \\
\hline 0.8 & NA & 9.74 & NA & -1.29 & NA & -3.61 & NA & -2.82 \\
\hline $\mathrm{IC}_{50}$ & & $8.90 \pm 0.82$ & & $66.21 \pm 6.24$ & & $52.10 \pm 4.43$ & & $61.09 \pm 6.06$ \\
\hline
\end{tabular}

NA: not affected.

samples from the genus Sargassum collected in Vietnam show three or four classes of Pol, ST, FFA, and TG $[4,23]$. In contrast to terrestrial plants with high content of $16 \mathrm{C}$ and 18C fatty acids, Lobophora sp. has low content of $16 \mathrm{C}$ and 18C fatty acids similar to brown seaweeds reported [4]. Particularly, lipid composition of Lobophora sp. contains a variety of fatty acids (23 types) including many long chains and 6 double bonds fatty acids belonging to $\omega 3(n-3)$ and $\omega 6$ (n-6) with $23.40 \%$ and $34.15 \%$ of the total FA, respectively. They have been considered to be beneficial for the human brain and overall health, including cardiovascular effects, improving the function of the heart and the liver, reducing blood pressure, anti-thrombosis, and helping with arthritis, cancer, and lung diseases [24-28]. Among those, $\alpha$ - and $\gamma$-linolenic acids (C18:3n-3-ALA and C18:3n-6-GLA) have the total content of $11.93 \%$, which are very important fatty acids in the formation and protection of the skin to potentially apply in the cosmetic industry [29].

Previous research has shown that the fatty acid composition in seaweed contains saturated fatty acids (SFAs) with high content and polyunsaturated fatty acids (MUFAs, PUFAs) with very low content. For example, the green algae Ulva rigida has $64.5 \%$ SFAs, $23.78 \%$ PUFAs, and $11.71 \%$ MUFAs [9]. The brown algae Padina pavonica has those with content of $43.45 \%, 1.7 \%$, and $23.67 \%$, respectively [30]; some red algae species belonging to Hypnea genus in Vietnam had the content of SFAs $>60 \%$, PUFAs from 3-12\%, and MUFAs is $8.55-27.88 \%$ [31]; other red algae have the similar fatty acid profile $[32,33]$. Interestingly, the fatty acid profile of this Lobophora sp. revealed the high level of polyunsaturated fatty acids (MUFAs, PUFAs) with the content of $75.10 \%$, which is extremely noteworthy to further study for potential application.

Lobophora sp. is an infrequent species that has been detected with 6 PUFAs belonging to the C20 fatty acid group including C20:2n-6; C20:3n-6 (DGLA); C20:3n-9; C20: 4n-3; C20:4n-6 (AA); C20:5n-3 (EPA), in which DGLA, AA and EPA have content of $1.12 \%, 12.14 \%$, and $11.56 \%$, respectively. They are precursors which provide the initial fatty acids for the biosynthesis process of eicosanoids. Those are signaling molecules that control many systems in the human body, of which the most important roles are involved in the inflammatory process, immune response, and the effect on the central nervous system [33-35].

Particularly, the fatty acid C22:6n-3 (DHA) has been identified in the total lipid of this species with the content of $14.26 \%$ which is a valuable fatty acid in foods that helps increase the brain development of children and young animals [36]. DHA content of this species is much higher than that of other brown seaweeds collected in Vietnam and of oysters, fish, and corals $[4,18,35]$. This can be a sign to distinguish seaweeds living in coral reefs from those living in coastal areas.

Recent researches on lipid usually have applied GC-MS, GC-FID techniques to detect the composition and content of fatty acids; however, fatty acids exist in both free and linked types. Nowadays, the application of HR-MS technique helps identify the hold of lipid molecules and even molecular species based on ion values of MS [17, 18, 21]. Some novel techniques such as LC-MS/MS have been applied to study the lipidome of seaweed [9, 10, 14, 15, 37]. With HPLCHRMS technique, 157 polar lipids molecular forms have been, for the first time, identified belonging to phospholipids, glycolipids, and betaine lipids of Lobophora sp.

Betaine lipids are an amphoteric subclass like phospholipids because they have a positively charged ammonium group. They are determined to have a function similar to phospholipids in many algae, fungi, and seedless plants [38-42]. DGTA is a glycerol-lipid that contains two fatty acids esterified with glycerol and an ether-linked polar group derived from an amino acid, while DGTS is an isomer structure and biosynthetic precursor of DGTA in the algae $[43,44]$. The coexistence of DGTS and DTGA in many types of algae can be explained by a partial conversion of DGTS to DGTA.

In addition to molecular species analysis, the total lipid from Lobophora sp. has been separated into polar lipid and unpolar lipid fractions, in which polar lipid contained phospholipid, betaine lipid, and glycolipid classes. In recent studies, the extracts from Lobophora species have been reported on such bioactivities as anti-oxidant, anti-inflammation, anti-microbial, and cytotoxic activities [3]. In this study, we have preliminarily screened the anti-inflammation activity of three lipid fractions through the NO production inhibition assay because $\mathrm{NO}$ is a key signaling molecule in the inflammation process [45]. The results show that the lipid fractions exhibited the strong NO production inhibition activity.

Particularly, the unpolar lipid fraction displays a higher NO inhibitory activity than polar lipid. This result contrasts with what has been reported on lipid fraction from a mud crab Scylla paramamosain, which shows the inhibition of the polar lipid higher than the unpolar lipid fraction [19]. This may be caused by the polar lipid content 
of Lobophora sp. which is lower than that of the mud crab S. paramamosain, $26.8 \%$ and $40.02 \%$, respectively. Other authors have demonstrated that an abundance of PUFAs in seaweed composition related to the display of antiinflammatory activity $[9,46]$. It has also been reported that anti-inflammatory activities $\left(\mathrm{IC}_{50}\right)$ of seafood range from 64.6 to $306.4 \mu \mathrm{g} / \mathrm{mL}$. In this report, the NO inhibition activity of lipid fractions is comparable to those of Octopus lipids [47] and sulfated polysaccharide of the marine brown algae Lobophora variegata [48]. Particularly, it is the first time that the Lobophora sp. lipid fractions have been demonstrated strong NO inhibition activity.

The recent reports indicate that the production of NO is increased by neuronal nitric oxide synthase (nNOS) mediated by the NF- $\kappa \mathrm{B}$ factor [49]. In addition, the transcription of Nf- $\kappa \mathrm{B}$ has been regulated by $n-3$ PUFAs [50]. Also, the report of Echeverria shows that n-3 PUFAs (especially EPA and DHA) partially reduce the proinflammatory indexes such as NF- $\kappa \mathrm{B}$ and Nrf2 [51]. Thus, it suggests that lipid fractions containing n-3 PUFAs may inhibit NO inhibition through regulation of factors NF- $\kappa \mathrm{B}$ and Nrf2.

In several expert studies, total extracts, their fractions, and molecular species have demonstrated bioactivities [52-56]. Particularly, glycolipids from different algae species have anti-viral, antibacterial, and anti-tumoral activity $[53,54]$. SQDG $(32: 0)$ and SQMG $(16: 0)$ are both described to have anti-microbial activity $[55,56]$. Some reports suggest that DGTS has the same function as PC due to their similar zwitterionic structure, and they are interchangeable in the cell [44]. It is significant that the MGDG displays anti-inflammatory activity and is combined with omega-3 to treat the regeneration of articular cartilage in the osteoarthritis adult. Thus, further study will be focusing on evaluating the bioactivities of the composition of Lobophora sp. lipid such as fatty acids, lipid classes, and molecular species.

\section{Conclusions}

In summary, lipid classes and fatty acid profile of seaweed Lobophora sp. have been well defined. The simultaneous detection of high-value long-chain polyunsaturated fatty acids such as AA, EPA, and DHA in this seaweed has inspired researches to study the origin of these fatty acids in the reef ecosystem. HPLC-HRMS technique has allowed identifying 157 molecular forms in polar lipid that are classified into betaine lipid, glycolipid, and phospholipid groups with 64,45 , and 48 molecular forms, respectively. The NO inhibition effects of lipid fractions including total lipid, polar lipid, and unpolar lipid have been reported for the first time with $\mathrm{IC}_{50}$ from 52.10 to $66.21 \mu \mathrm{g} / \mathrm{mL}$. These all suggest that Lobophora sp. lipids need to be further studied for potential application in food, medicine, and cosmetics.

\section{Data Availability}

No data were used to support this study.

\section{Conflicts of Interest}

The authors declare no conflicts of interest.

\section{Acknowledgments}

The authors would like to express their sincere thanks to the Laboratory of Comparative Biochemistry A. V. Zhirmunsky Institute of Marine Biology, Far-Eastern Branch of the Russian Academy of Sciences, 17 Palchevskogo str., Vladivostok 690041, Russian Federation. Furthermore, the authors are grateful to the Project "Researching and Assessing the Potential of Resources and the Ability to Exploit and Cultivate Seaweed in Economic Target Islands in Service of Socio-Economic Development" (Code: KC.09.05/ 16-20) for supporting their study.

\section{Supplementary Materials}

Figure 1S. HPLC-HRMS and fragmentations of PI $34: 1$ $\left[\mathrm{C}_{43} \mathrm{H}_{79} \mathrm{O}_{13} \mathrm{P}\right]^{-}$. (a) HPLC-HRMS chromatogram of $\left[\mathrm{C}_{43} \mathrm{H}_{79} \mathrm{O}_{13} \mathrm{P}\right]^{-}$. (b) Negative mass spectrometry $\left(\mathrm{MS}^{-}\right)$of $\left[\mathrm{C}_{43} \mathrm{H}_{79} \mathrm{O}_{13} \mathrm{P}\right]^{-}$. (c) Negative mass spectrometry $\left(\mathrm{MS}^{2-}\right)$ of signal at $\mathrm{m} / \mathrm{z}$ 835.5283. Figure 2S. HPLC-HRMS and fragmentations of PC 30:0 $\left[\mathrm{C}_{38} \mathrm{H}_{77} \mathrm{NO}_{8} \mathrm{P}\right]^{+}$. (a) HPLC-HRMS chromatogram of $\left[\mathrm{C}_{38} \mathrm{H}_{77} \mathrm{NO}_{8} \mathrm{P}\right]^{+}$. (b) Positive mass spectrometry $\left(\mathrm{MS}^{+}\right)$of signal at $\mathrm{m} / \mathrm{z}\left[\mathrm{C}_{38} \mathrm{H}_{77} \mathrm{NO}_{8} \mathrm{P}\right]^{+}$. (c) Negative mass spectrometry $\left(\mathrm{MS}^{-}\right)$of signal at $\mathrm{m} / \mathrm{z} 690.5025$ and $\mathrm{m} / \mathrm{z} 750.5026$. (c) Negative mass spectrometry $\left(\mathrm{MS}^{2-}\right)$ of signal at $\mathrm{m} / \mathrm{z}$ 690.5025. Figure 3S. HPLC-HRMS and fragmentations of PG $34: 2\left[\mathrm{C}_{40} \mathrm{H}_{74} \mathrm{O}_{10} \mathrm{P}\right]^{-}$. (a) HPLC-HRMS chromatogram of $[\mathrm{C} 40 \mathrm{H} 74 \mathrm{O} 10 \mathrm{P}]^{-}$. (b) Negative mass spectrometry $\left(\mathrm{MS}^{-}\right)$of $[\mathrm{C} 40 \mathrm{H} 74 \mathrm{O} 10 \mathrm{P}]^{-}$. (c) Negative mass spectrometry $\left(\mathrm{MS}^{2-}\right)$ of signal at $\mathrm{m} / \mathrm{z}$ 745.4985. Figure 4S. HPLC-HRMS and fragmentations of PA $40: 8$ $\left[\mathrm{C}_{43} \mathrm{H}_{68} \mathrm{O}_{8} \mathrm{P}\right]^{-}$. (a) HPLC-HRMS chromatogram of $[\mathrm{C} 43 \mathrm{H} 68 \mathrm{O} 8 \mathrm{P}]^{-}$. (b) Negative mass spectrometry $\left(\mathrm{MS}^{-}\right)$of $[\mathrm{C} 43 \mathrm{H} 68 \mathrm{O} 8 \mathrm{P}]^{-}$. (c) Negative mass spectrometry $\left(\mathrm{MS}^{2-}\right)$ of signal at $\mathrm{m} / \mathrm{z}$ 743.4609. Figure 5S. HPLC-HRMS and fragmentations of MGDG 38:9 $\left[\mathrm{C}_{47} \mathrm{H}_{72} \mathrm{O}_{10} \mathrm{Na}\right]^{+}$. (a) HPLCHRMS chromatogram of $[\mathrm{C} 47 \mathrm{H} 72 \mathrm{O} 10 \mathrm{Na}]^{+}$. (b) Positive mass spectrometry $\left(\mathrm{MS}^{+}\right)$of $[\mathrm{C} 47 \mathrm{H} 72 \mathrm{O} 10 \mathrm{Na}]^{+}$. (c) Negative mass spectrometry $\left(\mathrm{MS}^{-}\right)$of signal at $\mathrm{m} / \mathrm{z} 795.5046$ and $\mathrm{m} / \mathrm{z}$ 841.5021. (d) Positive mass spectrometry $\left(\mathrm{MS}^{2+}\right)$ of signal at $\mathrm{m} / \mathrm{z}$ 819.5023. Figure 6S. HPLC-HRMS and fragmentations of DGDG 34:1 $\left[\mathrm{C}_{49} \mathrm{H}_{89} \mathrm{O}_{15}\right]^{-}$. (a) HPLC-HRMS chromatogram of $[\mathrm{C} 49 \mathrm{H} 89 \mathrm{O} 15]^{-}$. (b) Negative mass spectrometry $\left(\mathrm{MS}^{-}\right)$of $[\mathrm{C} 49 \mathrm{H} 89 \mathrm{O} 15]^{-}$. (c) Negative mass spectrometry $\left(\mathrm{MS}^{-}\right)$of $[\mathrm{C} 49 \mathrm{H} 89 \mathrm{O} 15 \mathrm{Na}]^{+}$. (d) Negative mass spectrometry $\left(\mathrm{MS}^{2-}\right)$ of $\mathrm{m} / \mathrm{z}$ 917.600. Figure 7S. HPLCHRMS and fragmentations of SQDG $34: 1\left[\mathrm{C}_{43} \mathrm{H}_{79} \mathrm{O}_{12} \mathrm{~S}\right]^{-}$. (a) HPLC-HRMS chromatogram of $[\mathrm{C} 43 \mathrm{H} 79 \mathrm{O} 12 \mathrm{~S}]^{-}$. (b) Negative mass spectrometry $\left(\mathrm{MS}^{-}\right)$of $[\mathrm{C} 43 \mathrm{H} 79 \mathrm{O} 12 \mathrm{~S}]^{-}$. (c) Negative mass spectrometry $\left(\mathrm{MS}^{2-}\right)$ of signal at $\mathrm{m} / \mathrm{z}$ 819.5162. Figure 8S. HPLC-HRMS and fragmentations of DGTA 34:1 $\left[\mathrm{C}_{44} \mathrm{H}_{84} \mathrm{NO}_{7}\right]^{+}$. (a) HPLC-HRMS chromatogram of $[\mathrm{C} 44 \mathrm{H} 84 \mathrm{NO} 7]^{+}$. (b) Positive mass spectrometry $\left(\mathrm{MS}^{+}\right)$of $[\mathrm{C} 44 \mathrm{H} 84 \mathrm{NO}]^{+}$. (c) Positive mass spectrometry $\left(\mathrm{MS}^{2+}\right)$ of signal at $\mathrm{m} / \mathrm{z}$ 738.6298. Figure 9S. HPLC-HRMS 
and fragmentations of DGTS 28:0 $\left[\mathrm{C}_{38} \mathrm{H}_{74} \mathrm{NO}_{7}\right]^{+}$. (a) HPLC-HRMS chromatogram of [C38H74NO7] ${ }^{+}$. (b) Positive mass spectrometry $\left(\mathrm{MS}^{+}\right)$of $\left[\mathrm{C} 38 \mathrm{H} 74 \mathrm{NO}^{+}\right]^{+}$. (c) Positive mass spectrometry $\left(\mathrm{MS}^{2+}\right)$ of signal at $\mathrm{m} / \mathrm{z} 656.5459$. . (Supplementary Materials)

\section{References}

[1] A. Kumar, M. Memo, and A. Mastinu, "Plant behaviour: an evolutionary response to the environment?" Plant Biology, vol. 22, no. 6, pp. 961-970, 2020.

[2] A. Mahdavi, P. Moradi, and A. Mastinu, "Variation in terpene profiles of Thymus vulgaris in water deficit stress response," Molecules, vol. 25, no. 5, p. 1091, 2020.

[3] C. Vieira, J. Gaubert, O. De Clerck, C. Payri, G. Culioli, and O. P. Thomas, "Biological activities associated to the chemodiversity of the brown algae belonging to genus Lobophora (Dictyotales, Phaeophyceae)," Phytochemistry Reviews, vol. 16, no. 1, pp. 1-17, 2017.

[4] T. H. Pham, V. T. A. Nguyen, T. C. B. Nguyen, D. T. Dam, T. T. Le, and Q. L. Pham, Research on the Content of Lipid Classes and Fatty Acids from Sargassum Seaweed, in Proceedings of ASAM conferences in Vietnam, Hanoi, Vietnam, September 2017.

[5] K. J. Bowen, W. S. Harris, and P. M. Kris-Etherton, "Omega-3 fatty acids and cardiovascular disease: are there benefits?" Current Treatment Options in Cardiovascular Medicine, vol. 18, no. 11, pp. 18-69, 2016.

[6] K. H. M. Cardozo, T. Guaratini, M. P. Barros et al., "Metabolites from algae with economical impact," Comparative Biochemistry and Physiology Part C: Toxicology \& Pharmacology, vol. 146, no. 1-2, pp. 60-78, 2007.

[7] S. Eko, S. F. Akhmad, A. Masayuki, H. Masashi, and M. Kazuo, "Lipids, fatty acids, and fucoxanthin content from temperate and tropical brown seaweeds," Aquatic Procedia, vol. 7, pp. $66-75,2016$

[8] I. A. Guschina and J. L. Harwood, "Lipids and lipid metabolism in eukaryotic algae," Progress in Lipid Research, vol. 45, no. 2, pp. 160-186, 2006.

[9] D. Lopes, A. S. P. Moreira, F. Rey et al., "Lipidomic signature of the green macroalgae Ulva rigida farmed in a sustainable integrated multi-trophic aquaculture," Journal of Applied Phycology, vol. 31, no. 2, pp. 1369-1381, 2019.

[10] E. Costa, P. Domingues, T. Melo et al., "Lipidomic signatures reveal seasonal shifts on the relative abundance of high-valued lipids from the Brown algae fucus vesiculosus," Marine Drugs, vol. 17 , no. 6 , p. 335, 2019.

[11] H. He, R. P. Rodgers, A. G. Marshall, and C. S. Hsu, "Algae polar lipids characterized by online liquid chromatography coupled with hybrid linear quadrupole ion trap/fourier transform ion cyclotron resonance mass spectrometry," Energy \& Fuels, vol. 25, no. 10, pp. 4770-4775, 2011.

[12] I. Naumann, K. H. Darsow, C. Walter, H. A. Lange, and R. Buchholz, "Identification of sulfoglycolipids from the alga Porphyridium purpureum by matrix-assisted laser desorption/ionisation quadrupole ion trap time-of-flight mass spectrometry," Rapid Communications in Mass Spectrometry, vol. 21, no. 19, pp. 3185-3192, 2007.

[13] P. Kumari, M. Kumar, C. R. K. Reddy, and B. Jha, "Nitrate and phosphate regimes induced lipidomic and biochemical changes in the intertidal macroalga Ulva lactuca (Ulvophyceae, Chlorophyta)," Plant and Cell Physiology, vol. 55, no. 1, pp. 52-63, 2014.
[14] T. Melo, E. Alves, V. Azevedo et al., "Lipidomics as a new approach for the bioprospecting of marine macroalgaeunraveling the polar lipid and fatty acid composition of Chondrus crispus," Algal Research, vol. 8, pp. 181-191, 2015.

[15] E. Costa, T. Melo, A. S. P. Moreira et al., "Decoding bioactive polar lipid profile of the macroalgae Codium tomentosum from a sustainable IMTA system using a lipidomic approach," Algal Research, vol. 12, pp. 388-397, 2015.

[16] E. G. Bligh and W. J. Dyer, "A rapid method of total lipid extraction and purification," Canadian Journal of Biochemistry and Physiology, vol. 37, no. 1, pp. 911-917, 1959.

[17] T. P. L. Nguyen, V. T. A. Nguyen, T. T. T. Do, Q. T. Nguyen, Q. L. Pham, and T. T. Le, "Fatty acid composition, phospholipids molecules and bioactivities of lipids of the mud crab Scylla paramamosain," Journal of Chemistry, vol. 2020, Article ID 8651453, 9 pages, 2020.

[18] Q. T. Tran, T. T. T. Le, M. Q. Pham et al., "Fatty acid, lipid classes and phospholipid molecular species composition of the marine clam Meretrix lyrata (Sowerby 1851) from Cua Lo beach, Nghe an province, Vietnam," Molecules, vol. 24, pp. 895-901, 2019.

[19] S. Harrabi, W. Herchi, H. Kallel, P. Mayer, and S. Boukhchina, "Liquid chromatographic-mass spectrometric analysis of glycerophospholipids in corn oil," Food Chemistry, vol. 114, no. 2, pp. 712-716, 2009.

[20] S. Boukhchina, K. Sebai, A. Cherif, H. Kallel, and P. M. Mayer, "Identification of glycerophospholipids in rapeseed, olive, almond, and sunflower oils by LC-MS and LC-MS-MS," Canadian Journal of Chemistry, vol. 82, no. 7, pp. 1210-1215, 2004.

[21] A. B. Imbs, V. G. Rybin, V. I. Kharlamenko et al., "Polyunsaturated molecular species of galactolipids: markers of zooxanthellae in a symbiotic association of the soft coral Capnella sp. (Anthozoa: Alcyonacea)," Russian Journal of Marine Biology, vol. 41, no. 6, pp. 461-467, 2015.

[22] T. Mosmann, "Rapid colorimetric assay for cellular growth and survival: application to proliferation and cytotoxicity assays," Journal of Immunological Methods, vol. 65, no. 1-2, pp. 55-63, 1983.

[23] B. J. Gosch, M. Magnusson, N. A. Paul, and R. De Nys, “Total lipid and fatty acid composition of seaweeds for the selection of species for oil-based biofuel and bioproducts," GCB Bioenergy, vol. 4, no. 6, pp. 919-930, 2012.

[24] P. J. Gillies, W. S. Harris, and P. M. Kris-Etherton, “Omega-3 fatty acids in food and pharma: the enabling role of biotechnology," Current Atherosclerosis Reports, vol. 13, no. 6, pp. 467-473, 2011.

[25] D. Mozaffarian and J. H. Y. Wu, "Omega-3 fatty acids and cardiovascular disease," Journal of the American College of Cardiology, vol. 58, no. 20, pp. 2047-2067, 2011.

[26] H. Poudyal, S. K. Panchal, L. C. Ward, and L. Brown, "Effects of ALA, EPA and DHA in high-carbohydrate, high-fat dietinduced metabolic syndrome in rats," The Journal of Nutritional Biochemistry, vol. 24, no. 6, pp. 1041-1052, 2013.

[27] G. P. Eckert, U. Lipka, and W. E. Muller, "Omega-3 fatty acids in neurodegenerative diseases: focus on mitochondria," Prostaglandins, Leukotrienes and Essential Fatty Acids, vol. 88, no. 1, pp. 105-114, 2013.

[28] Z.-Y. Liu, D.-Y. Zhou, Z.-X. Wu et al., "Extraction and detailed characterization of phospholipid-enriched oils from six species of edible clams," Food Chemistry, vol. 239, pp. 11751181, 2018.

[29] T.-H. Huang, P.-W. Wang, S.-C. Yang, W.-L. Chou, and J.-Y. Fang, "Cosmetic and therapeutic applications of fish oil's 
fatty acids on the skin," Marine Drugs, vol. 16, no. 8, p. 256, 2018.

[30] G. Bernardini, M. Minetti, G. Polizzotto, M. Biazzo, and A. Santucci, "Pro-apoptotic activity of French polynesian Padina pavonica extract on human osteosarcoma cells," Marine Drugs, vol. 16, no. 12, p. 504, 2018.

[31] T. T. Le, V. T. A. Nguyen, T. H. Nguyen et al., "Survey lipid content and composition of fatty acids from Hypnea seaweed," Vietnam Journal of Chemistry, vol. 51, pp. 49-53, 2013.

[32] B. D. A. Daniel, C. D. Jaécio, A. S. R. Simone et al., "Fatty acid composition from the marine red algae Pterocladiella capillacea (S. G. Gmelin) Santelices \& Hommersand 1997 and Osmundaria obtusiloba (C. Agardh) R. E. Norris 1991 and its antioxidant activity," Anais da Academia Brasileira de Ciências, vol. 90, pp. 449-459, 2018.

[33] T. T. Le, Filter research, isolation, and identification active fatty acid, arachidonic acid and prostaglandin from red seaweed, PhD thesis in Chemistry, Archived at GUST, VAST, 2015.

[34] S. V. Khotimchenko and V. E. Vaskovsky, "Distribution of C20 polyenoic fatty acids in red macrophytic algae," Botanica Marina, vol. 33, pp. 525-528, 1990.

[35] T. P. L. Dang, Study on lipid composition and molecular forms of phospholipids from some soft corals in Vietnam, $\mathrm{PhD}$ thesis in Chemistry, Archived at GUST, VAST, 2016.

[36] Q. L. Pham and V. M. Chau, Biologically Active Lipids and Fatty Acids from Naturepp. 100-213, Science and Technology Publising House, Hanoi, Vietnam, 1 edition, 2005.

[37] E. Costa, T. Melo, A. S. P. Moreira et al., "Valorization of lipids from Gracilaria sp. through lipidomics and decoding of antiproliferative and anti-inflammatory activity," Marine Drugs, vol. 15, pp. 1-17, 2017.

[38] W. Eichenberger and M. Hofmann, "Metabolism and distribution of betaine lipids in algae," Metabolism, Structure and Utilization of Plant Lipids", pp. 18-21, Centre National Pédagogique, Montffeury, Tunesie, 1992.

[39] V. E. Vaskovsky, S. V. Khotimchenko, and E. M. Boolugh, "Distribution of diacylglycerotrimethylhomoserine and phosphatidylcholine in mushrooms," Phytochemistry, vol. 47, no. 5, pp. 755-760, 1998.

[40] R. M. Klug and C. Benning, "Two enzymes of diacylglyceryl$\mathrm{O}-4$ '-(N,N,N,-trimethyl)homoserine biosynthesis are encoded by btaA and btaB in the purple bacterium Rhodobacter sphaeroides," Proceedings of the National Academy of Sciences, vol. 98, no. 10, pp. 5910-5915, 2001.

[41] W. R. Riekhof, C. Andre, and C. Benning, "Two enzymes, $\mathrm{BtaA}$ and $\mathrm{BtaB}$, are sufficient for betaine lipid biosynthesis in bacteria," Archives of Biochemistry and Biophysics, vol. 441, no. 1, pp. 96-105, 2005.

[42] O. A. Rozenstvet, S. V. Saksonov, V. R. Filin, and V. M. Dembitsky, "Seasonal changes of lipid content in the leaves of some ferns," Physiologia Plantarum, vol. 113, no. 1, pp. 59-63, 2001.

[43] K. Künzler and W. Eichenberger, "Betaine lipids and zwitterionic phospholipids in plants and fungi," Phytochemistry, vol. 46, no. 5, pp. 883-892, 1997.

[44] A. Vieler, C. Wilhelm, R. Goss, R. Süß, and J. Schiller, "The lipid composition of the unicellular green alga Chlamydomonas reinhardtii and the diatom Cyclotella meneghiniana investigated by MALDI-TOF MS and TLC," Chemistry and Physics of Lipids, vol. 150, no. 2, pp. 143-155, 2007.

[45] J. N. Sharma, A. Al-Omran, and S. S. Parvathy, "Role of nitric oxide in inflammatory diseases," Inflammopharmacology, vol. 15, no. 6, pp. 252-259, 2007.
[46] A. H. Banskota, R. Stefanova, S. Sperker, R. Melanson, J. A. Osborne, and S. J. B. O’Leary, "Five new galactolipids from the freshwater microalga Porphyridium aerugineum and their nitric oxide inhibitory activity," Journal of Applied Phycology, vol. 25, no. 4, pp. 951-960, 2013.

[47] T. Ahmad, D. Rudd, M. Kotiw, L. Liu, and K. Benkendorff, "Correlation between fatty acid profile and anti-inflammatory activity in common Australian seafood by-products," Marine Drugs, vol. 17, no. 3, pp. 155-174, 2019.

[48] R. C. L. Siqueira, M. S. J. da Silva, A. d. F. Pires et al., "In vivo anti-inflammatory effect of a sulfated polysaccharide isolated from the marine brown algae Lobophora variegata," Pharmaceutical Biology, vol. 49, no. 2, pp. 167-174, 2011.

[49] G. Rathnasamy, V. Sivakumar, P. Rangarajan, W. S. Foulds,

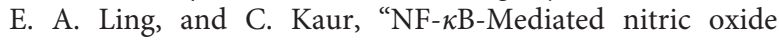
production and activation of caspase- 3 cause retinal ganglion cell death in the hypoxic neonatal retina," Investigative Opthalmology \& Visual Science, vol. 55, no. 9, pp. 5878-5889, 2014.

[50] R. Valenzuela and L. A. Videla, "Impact of the Co-administration of $\mathrm{N}-3$ fatty acids and olive oil components in preclinical nonalcoholic fatty liver disease models: a mechanistic view," Nutrients, vol. 12, no. 2, pp. 499-512, 2020.

[51] F. Echeverría, R. Valenzuela, A. Espinosa et al., "Reduction of high-fat diet-induced liver proinflammatory state by eicosapentaenoic acid plus hydroxytyrosol supplementation: involvement of resolvins RvE1/2 and RvD1/2," The Journal of Nutritional Biochemistry, vol. 63, pp. 35-43, 2019.

[52] A. K. Gupta, M. A. Ratherme)"[?--]>, A. Kumar Jha et al., "Artocarpus lakoocha Roxb. and Artocarpus heterophyllus Lam. flowers: new sources of bioactive compounds," Plants, vol. 9, no. 10, p. 1329, 2020.

[53] E. Plouguerné, B. A. P. Da Gama, R. C. Pereira, and E. Barreto-Bergter, "Glycolipids from seaweeds and their potential biotechnological applications," Frontiers in Cellular and Infection Microbiology, vol. 4, pp. 1-5, 2014.

[54] J. W. Blunt, B. R. Copp, R. A. Keyzers, M. H. G. Munro, and M. R. Prinsep, "Marine natural products," Natural Product Reports, vol. 33, no. 3, pp. 382-431, 2016.

[55] F. K. El Baz, G. S. El Baroty, H. H. Abd El Baky, O. I. Abd El Salam, and E. A. Ibrahim, "Structural characterization and biological activity of sulfolipids from selected marine algae," Grasas y Aceites, vol. 64, pp. 561-571, 2013.

[56] H. Wang, Y. L. Li, W. Z. Shen, W. Rui, X. J. Ma, and Y. Z. Cen, "Antiviral activity of a sulfoquinovosyldiacylglycerol (SQDG) compound isolated from the green alga Caulerpa racemosa," Botanica Marina, vol. 50, pp. 185-190, 2007. 